\title{
Experimental Study on Emulsion Formation in Buoyancy-Driven Microfluidics Devices
}

\author{
Yingying Chen ${ }^{1}$, Cheng $\mathrm{Yu}^{2}$, Xiangdong Liu ${ }^{1}$, Liangyu $\mathrm{Wu}^{1}$ \\ ${ }^{1}$ School of Hydraulic, Energy and Power Engineering, Yangzhou University \\ Yangzhou 225127, China \\ yychen@microflows.net; liuxd@yzu.edu.cn; lywu@yzu.edu.cn \\ ${ }^{2}$ School of Energy and Environment, Southeast University \\ Nanjing 210096, China \\ iamyucheng@seu.edu.cn
}

\begin{abstract}
Emulsion formation in a buoyancy-driven microfluidics device is studied experimentally via a visualization system comprised of a high-speed CCD and a microscope. The typical droplet formation regimes and interfacial behaviors during droplet formation is investigated. Variation in the droplet size and formation regime transition caused by the flow rate of the dispersed phase is discussed. The results indicate that the emulsions produced through the dripping regime when the flow of the dispersed phase is relatively slow are highly monodispersed. The jetting regime and transitional regime can produce emulsions under higher frequency comparing with dripping regime. However, the droplets are polydispersed and less control can be achieved.
\end{abstract}

Keywords: emulsions; microfluidics; interface; control; buoyancy-driven

\section{Introduction}

Emulsions are widely used in many fields, such as inertia confinement fusion [1, 2], biomedical engineering [3], material engineering [4] and chemical reaction [5]. Generating and manipulating emulsions through microfluidic approaches has great adaptability, designability and controllability and has drawn extensive attention.

Buoyancy-driven microfluidic device in unconfined space is a special type of microfluidic device [5], in which large density difference exists between the dispersed phase and the stationary continuous phase. The emulsion formation process is mainly affected by buoyancy (gravity), interfacial tension and inertia force of the dispersed phase. Buoyancy-driven microfluidics, with fewer dominating forces and interferences involved in the emulsion formation process, are preferable in producing monodispersed emulsions [6]. Though the dropping regime in buoyancy-driven microfluidics devices has been reported in current researches, other regimes as well as the regime transition requires further investigation. Therefore, a visualization experiment is carried out to further study the droplet formation platform in the buoyancy-driven microfluidic device. Typical formation regimes and interfacial behaviours during droplet formation are investigated. Variation in the droplet size and formation regime caused by the flow rate of the dispersed phase is discussed.

\section{Experimental system}

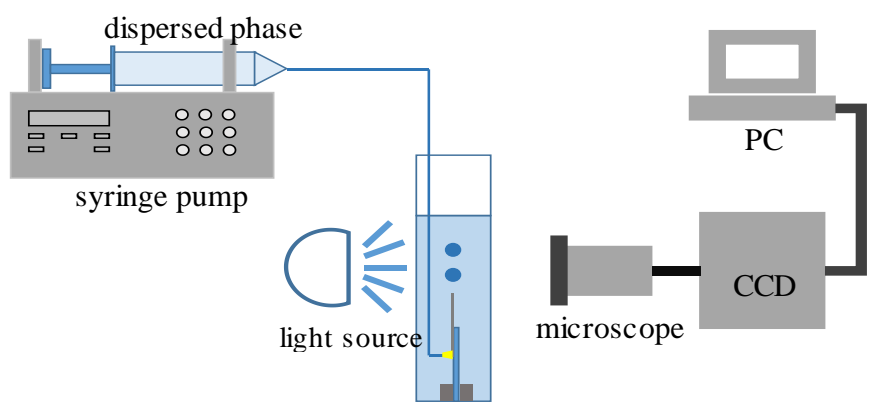

Fig. 1: Experimental setup. 
As shown in Figure 1, the buoyancy-driven microfluidics device used in the preparation of the single emulsion was placed in the bottom of a liquid pool filled with continuous phase, and the dispersed phase was injected by a syringe pump. A CCD was connected with a microscope to record the droplet formation processes. The operation temperature was $22 \pm 2{ }^{\circ} \mathrm{C}$. Three times of the experiments have been conducted to achieve the reliable results with strict control over the experimental flow conditions.

\section{Experimental results and discussion}

\subsection{Typical formation regimes}

Two typical formation regimes are distinguished in the buoyancy-driven microfluidics device: dripping and jetting. Transitional regime with characteristics of both dripping and jetting is observed as well.

Figure 2 shows the formation of a droplet under a typical dripping regime which can be divided into two stages: growth and detaching. The growth stage accounts for about $91 \%$ of the total droplet formation time. Interfacial tension dominants during growth stage that the dispersed phase is completely attached to the nozzle. With further injection of the dispersed phase, the interface shape deviates from spherical indicating that buoyancy is competing with interfacial tension in the detaching stage. A neck is formed between the dispersed phase droplet and the outlet whose diameter decreases drastically with time. The neck breaks and the droplet rises inside the pool under buoyancy.

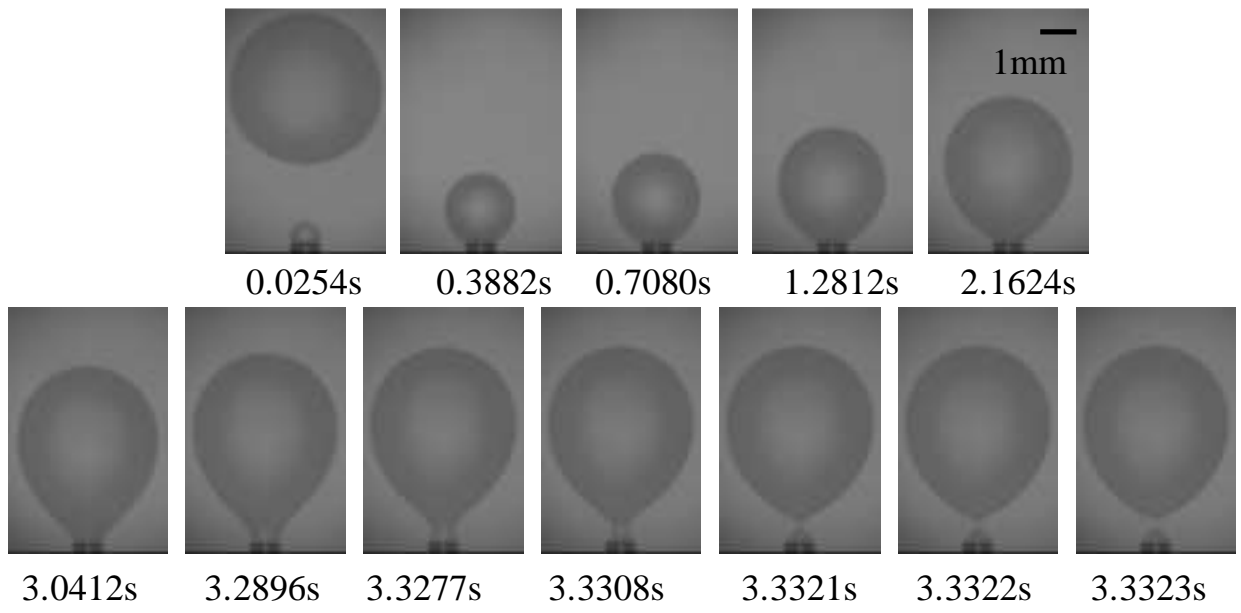

Fig. 2: Dripping regime.

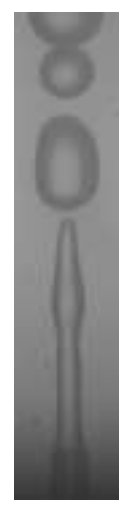

Oms
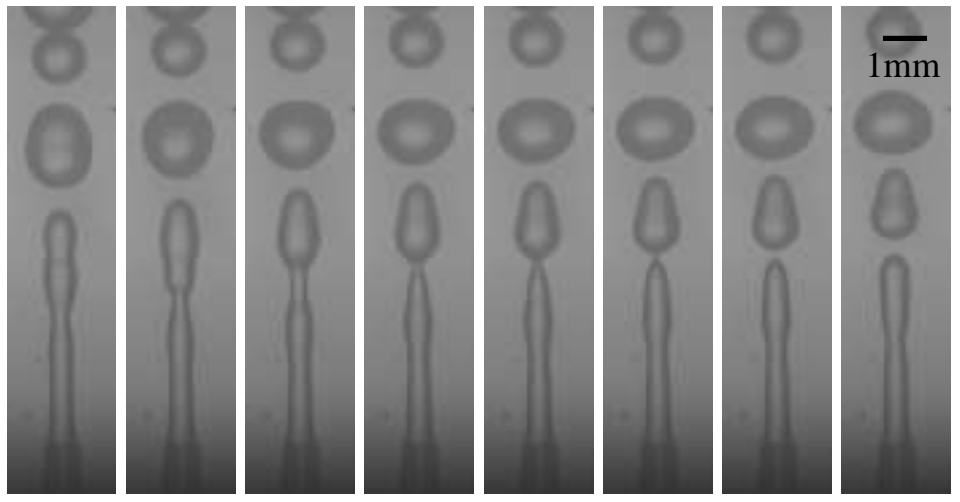

Fig. 3: Jetting regime.

Jetting regime occurs under high flow rate of the dispersed phase due to high inertia force from the dispersed phase as shown in figure 3. A cycle of droplet formation under jetting is much shorter than dripping. Differing from traditional microfluidics devices, no narrowing jetting dominated by viscous force of the continuous phase is observed. Undulations 
on the interface caused by capillary waves are observed. The fastest developing capillary waves eventually lead to the fracture of the jet.

A typical transitional regime with the characteristics of both dripping and jetting is shown in figure 4 . The interface is stretched in the vertical direction due to the inertia force. The volume of the dispersed phase attached to the nozzle is larger than that of the dripping regime after the detachment of the previous droplet. The time required for the droplet to grow to the critical volume of detaching is less which accounts for about $50 \%$ of the total droplet formation time. Two times of neck shrinking are observed during the detaching stage due to the competition between the interfacial tension and inertia force.

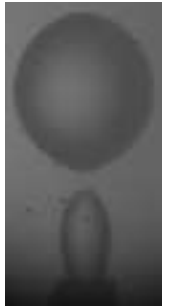

$0.185 \mathrm{~ms}$

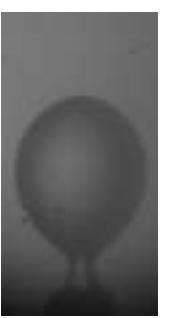

$94.444 \mathrm{~ms}$

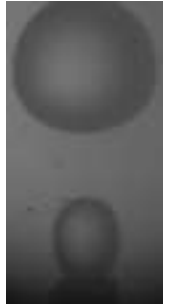

$5.926 \mathrm{~ms}$
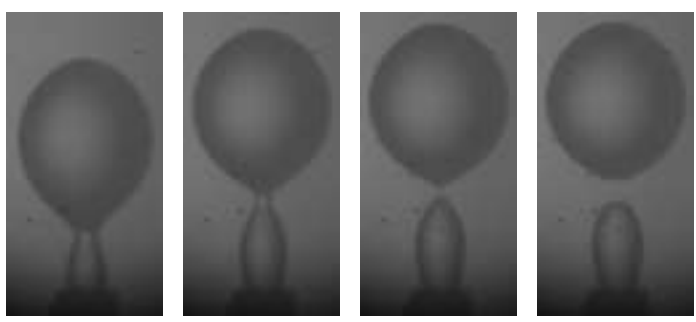

$90.791 \mathrm{~ms}$

Fig. 4: Transitional regime.

\subsection{Analysis of influencing factors}

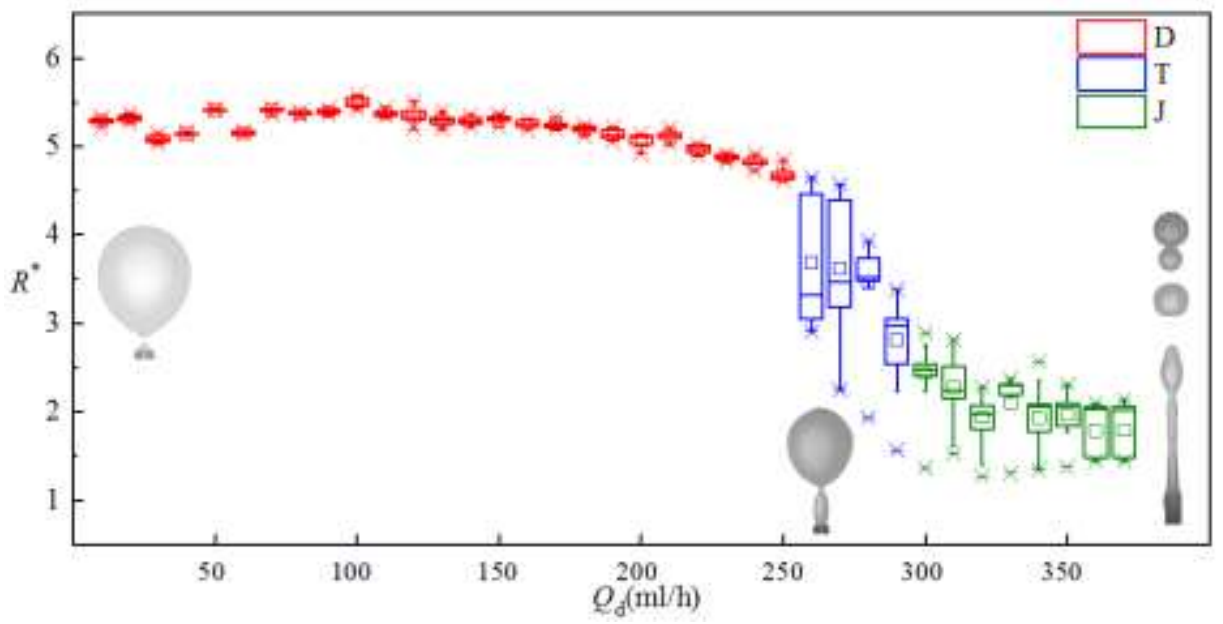

Fig. 5: Variation of droplet size and generation formation regime with the flow rate of the dispersed phase $Q_{\mathrm{d}}$

Figure 5 shows the droplet size and the generation formation regime as a function of the flow rate of dispersed phase in which D represents dripping regime, T represents transitional regime, and $\mathrm{J}$ represents jetting regime. $Q_{\mathrm{d}}$ has little effect on the droplet size $R^{*}$ under dripping regime and emulsions generated are highly monodispersed. The transition from dripping regime to transitional regime occurs when $Q_{\mathrm{d}}>255 \mathrm{ml} / \mathrm{h}$. The monodispersity of droplets prepared within this flow range deteriorated dramatically due to alternate generation of big and small droplets. $Q_{\mathrm{d}}$ has strong influence on the size of the droplets in transitional regime, and the minimum size of the droplets varie discontinuously. The transitional regime occupies 
relatively small flow rate range. When $Q_{\mathrm{d}}$ exceeds $295 \mathrm{ml} / \mathrm{h}$, jetting occurs. Variation in droplet size due to increasing in $Q_{\mathrm{d}}$ is reduced when the inertia force completely dominants the jetting regime.

\section{Conclusion}

Emulsion formation in a buoyancy-driven microfluidics device is studied experimentally via a visualization system comprised of a high-speed CCD and a microscope in this paper. Typical formation regimes and interfacial behaviors during droplet formation are investigated. Variation in the droplet size and formation regime caused by the flow rate of the dispersed phase is discussed. The conclusions can be summarized as:

(1) Dripping is preferable in producing highly monodispserd emulsions while jetting is suitable for applications that require fast emulsions generation.

(2) Increasing the flow rate of the dispersed phase leads to the transition from dripping regime to transitional regime and finally to jetting regime. The dominant force changes from interfacial tension to inertia force, accompanied by sharp variation in droplet size and deterioration in the monodispersity of droplets.

\section{Acknowledgements}

This work is supported by National Natural Science Foundation of China (No.51706194 and U1530260).

\section{References}

[1] A. A. Al-Abidi, S. Mat, K. Sopian, M. Y. Sulaiman, A. T. Mohammad, "Numerical study of PCM solidification in a triplex tube heat exchanger with inner and external fins," Int. J. Heat Mass Transfer, vol. 61, pp. 684-695, 2013.

[2] N. H. S. Tay, F. Bruno, M. Belusko, "Comparison of pinned and finned tubes in a phase change thermal energy storage system using CFD," Appl. Energ., vol. 104, pp. 79-86, 2013.

[3] C. Z. Ji, Z. H. Qin, Z. Low, S. Dubey, F. H. Choo, F. Duan, "Non-uniform heat transfer suppression to enhance PCM melting by angled fins," Appl. Therm. Eng., vol. 129 pp. 269-279, 2018.

[4] A. M. Ibrahem, M. F. El-Amin, S. Y. Sun, "Effects of nanoparticles on melting process with phase-change using the lattice Boltzmann method," Results in Physics, vol. 7, pp. 1676-1682, 2017.

[5] A. S. Chaurasia, D. N. Josephides, S. Sajjadi, "Buoyancy-driven drop generation via microchannel revisited," Microfluidics and Nanofluidics, vol. 18, no. 5-6, pp. 943-953, 2015.

[6] Y. E. Milian, A. Gutierrez, M. Grageda, S. Ushak, "A review on encapsulation techniques for inorganic phase change materials and the influence on their thermophysical properties," Renewable and Sustainable Energy Reviews, vol. 73, pp. 983-999, 2017. 